\title{
Review: soft drink consumption is associated with increased energy intake and body weight
}

Vartanian LR, Schwartz MB, Brownell KD. Effects of soft drink consumption on nutrition and health: a systematic review and metaanalysis. Am J Public Health 2007;97:667-75.

\section{$Q$ Is soft drink consumption associated with increased energy intake, increased body weight, displacement of nutrients, and an increased risk of chronic disease?}

\section{METHODS}

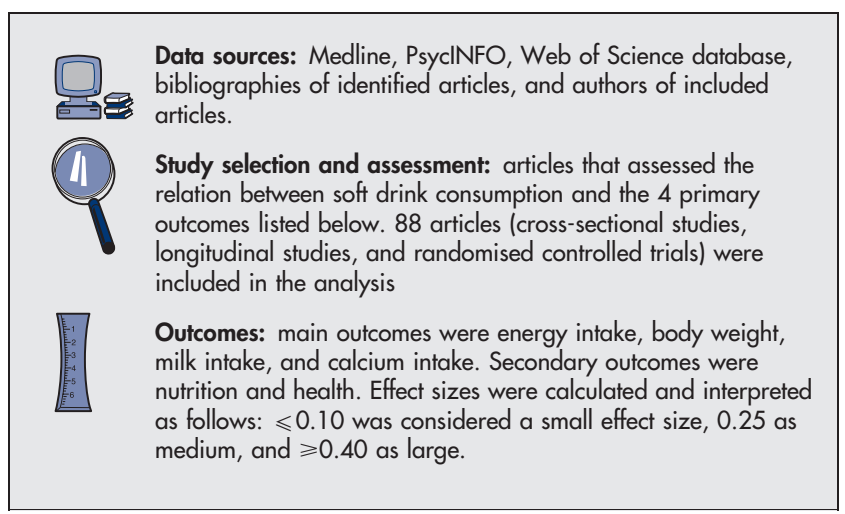

\section{MAIN RESULTS}

Only the results of meta-analyses of randomised controlled trials and longitudinal studies are reported here. Soft drink consumption was associated with increased energy intake and body weight and reduced milk and calcium intake (table). The associations between soft drink consumption and intake of various nutrients and various health conditions were not presented by study type and so are not reported here.

\section{CONCLUSION}

Soft drink consumption is associated with increased energy intake and body weight and reduced milk and calcium intake. For correspondence: Dr K D Brownell, Yale University, New Haven, CT, USA. kelly.brownell@yale.edu

Source of funding: Rudd Foundation.

\section{Commentary}

7 he review by Vartanian et al adds to our knowledge of the negative effects of soft drink consumption on nutrition and health. Overall, this review of 88 randomised controlled trials, longitudinal, and crosssectional studies was strong. The authors considered variables such as funding sources, and the results of the review are strengthened by greater associations found among those studies with more robust designs. Limitations of the review include failure to provide readers with a table of characteristics of the included studies and a description of how the quality of these studies was assessed. In addition, there remained significant heterogeneity in the effect sizes observed, despite performing metaanalysis by different design and moderating variables. The findings regarding nutrient intake and health outcomes are further limited by the small number of included studies. For example, the conclusion of an association between soft drink consumption and type 2 diabetes is based on a single, albeit large, cohort study.

Vartanian et al used effect size as a measure of association; the closer the effect size to 1.0 or -1.0 , the larger the positive or negative association. The results indicate positive associations between soft drink consumption and energy intake and body weight and negative associations with milk and calcium intake. The recommendation by Vartanian et al for reduced soft drink consumption remains within the scope of their review and follows from the strongest finding of their review: the greater the soft drink consumption, the greater the food energy intake. Using clinical expertise, most practitioners would agree that the benefits of limiting soft drink consumption outweigh the risks. Thus, the review by Vartanian et al provides practitioners with evidence to recommend limiting soft drink consumption to their clients.

Jennifer Yost, RN, MA

New York University College of Nursing New York, NY, USA

Associations between soft drink consumption and various outcomes*

\begin{tabular}{llc}
\hline Outcomes & Number and type of studies & Mean effect size (p value) \\
\hline Energy intake & 5 longitudinal studies & $0.24(p<0.001)$ \\
& 4 long-term randomised controlled trials (consumption over 3-10 wks) & $0.30(p<0.001)$ \\
Body weight & 12 short-term randomised controlled trials (consumption over a meal or single day) & $0.21(p=0.004)$ \\
Milk intake & $\{10\}$ longitudinal studies & $0.09(p<0.001)$ \\
Calcium intake & 7 randomised controlled trials & $0.24(p<0.001)$ \\
& 5 longitudinal studies & $-0.21(p<0.001)$ \\
\end{tabular}

*Effect sizes $\leqslant 0.10$ were considered to be small, 0.25 to be medium, and $\geqslant 0.40$ to be large.

tInformation provided by author. 